\title{
1 Solubilization of lignin in copolymer micelles in aqueous solution
}

2 Mohammadali Azadfar ${ }^{\mathrm{a}^{*}}$, William C. Hiscox ${ }^{\mathrm{b}}$ and Shulin Chen ${ }^{\mathrm{a}^{* *}}$

a. Department of Biological Systems Engineering; b. Center for NMR spectroscopy, Department of Chemistry; Washington State University, Pullman, WA 99164-6120, USA

5 ABSTRACT: The solubilization of lignin in self-aggregating triblock amphiphilic poly(ethylene oxide)-poly(propylene oxide)-poly(ethylene oxide) (PEO-PPO-PEO) in aqueous solution has

7 been studied utilizing liquid-state proton nuclear magnetic resonance spectroscopy $\left({ }^{1} \mathrm{H}\right.$ NMR) and diffusion-ordered nuclear magnetic resonance spectroscopy (DOSY NMR). Our hypothesis

9 for copolymer micelle incorporation of lignaceous species includes a "hydrophilic-aromatic interaction model", in which lignin derivatives with amphiphilic characteristics, known to be abundant in the effluent from pulp mill and pretreatment of various lignocellulosic materials,

12 have an affinity for these micelles, and tend to form relatively organized structures within them.

13 Here we show how the chemical/structural features of lignin units and small molecule lignin 14 models determine their solubilization behavior in the micelles. It was found that a buildup of concentration series of lignin and lignin-based model compounds guaiacol, eugenol, phenol, guaiacylglycerol-beta-guaiacyl ether, ferulic acid in Pluronic ${ }^{\circledR}$ F68-deuterium oxide solution

17 results in significant upfield chemical shifts of $\mathrm{PEO}-\left(\mathrm{CH}_{2}-\mathrm{CH}_{2}\right)$ and $\mathrm{PPO}-\left(\mathrm{CH}_{3}\right)$ proton resonances, and at a critical guest concentration (CGC), dramatic upfield shifts due to gross

19 structural transitions in the micelles. We present evidence that copolymer micelle-lignin interactions depend on both chemical functional group characteristics of solute (i.e., polarity, $\mathrm{H}$ -

21 bonding ability) and $\pi-\pi$ interactions between aromatic/conjugated groups. Our results

22 demonstrate how the loci of incorporated solute in the block copolymer micelles are affected by 23 these features. 
24 Keywords: Lignin; Block copolymer; Micelle; Solubilization

\section{Introduction}

Block copolymers comprise more than one type of block. Usually, the properties of the blocks within the copolymer are quite different from one another and this results in spontaneous self assembly in aqueous solution [1]. One of the key determinants of block copolymers, hydrophiliclipophilic balance (HLB), predicts the formation of aggregates at the critical micelle concentration (CMC) in aqueous media. Such aggregates or micelles may have spherical, cylindrical, rod-shaped or lamellar shapes [2]. In polar solvents such as water, the hydrophobic blocks are incorporated in the core and the hydrophilic blocks, along with water, form the shell. Due to the polarity gradient present in micelles, the aggregates are able to accommodate hydrophobic substances and thus enhance the solubility of these materials, which are normally relatively insoluble substances in aqueous solutions [3, 4].

Solubilization phenomena in aqueous and nonaqueous solutions of conventional low molecular weight surfactants have been extensively investigated theoretically $[1,5]$ and experimentally $[6$, 7]. However, block copolymers have shown remarkable potential compared to conventional low molecular weight surfactants because of their micellization ability in a variety of solvents [8]. Block copolymers are utilized in diverse applications, such as delivery of drugs, as scavengers for pollutants, as chemical extraction aids in separation processes, and for micellar catalysis [911]. Block copolymer architecture (ABA, BAB, ABC, AB, BA), hydrophobic-lipophilic balance (HLB), and discrete block molecular weights can significantly influence their solubilization capacity [12]. Many low molecular weight solubilizates (guest/solute), including aromatic solvents benzene, toluene, naphthalene, pyrene, and anthracene, have been investigated in block copolymers $[7,13]$. To harness the benefit of block copolymer solubilization phenomena, it is 
47 necessary to understand how the extent of solubilization can be affected by the structural

48 properties of the solubilizate and the block copolymer.

49 Hydrophobic and hydrophilic interactions between the solubilizate and the blocks that form the 50 core and shell of micelles control the scope of solubilization. For instance, the solubilization of 51 gallates in polymeric micelles has been studied by Heins and coworkers [14]. Using electron spin 52 resonance spectroscopy (ESR) and nuclear magnetic resonance spectroscopy (NMR), the authors 53 reported that the locations of antioxidant gallates in micelles are dependent on their 54 hydrophobicity. Also, aromatic molecules were found to be solubilized preferentially compared 55 to aliphatic hydrocarbons in A-B-A type block copolymers [5]. Furthermore, experimental 56 results for hydrocarbon solutes in polyethylene oxide (PEO)-polypropylene oxide (PPO) block 57 copolymers demonstrated large solubilization capacities and high selectivity for aromatic over 58 aliphatic solutes in block copolymer micelles, compared to conventional low molecular weight 59 surfactant micelles [13]. The molecular volume of the solutes can also influence the amount of 60 solubilization in micelles. It has been observed that solutes with larger size are solubilized in 61 smaller number [15]. However, given identical molecular volumes, the polarity of the 62 compounds controls the selectivity of solubilization $[7,16]$. It was noted that the solubility of 63 benzene in polar and non-polar blocks of PEO/PPO was significantly larger compared to n64 hexane in the entire composition of the block copolymer [7]. Lignin, with its combination of 65 aromatic and aliphatic hydrocarbons, having polar functional groups, aliphatic bridges and a 66 variety of oxygenated moieties, can show a unique solubilization pattern in aqueous dispersions 67 of block copolymer micelles. Lignin is often described as a random polymer of three mono68 lignols, $\rho$-coumaryl (H), coniferyl (guiacyl, G) and sinapyl (S) alcohols [17]. 
69 In this study, lignin is obtained from black effluent produced by the sequential ozone and 70 soaking aqueous ammonia pretreatment of wheat straw, and was previously characterized as 71 partially-depolymerized fragments of $\mathrm{H}-\mathrm{G}-\mathrm{S}$ with $\mathrm{S} / \mathrm{G}$ ratio of 0.35 and average molecular 72 weight of $750(\mathrm{~g} / \mathrm{mol})$ [18]. Lignin is partially decomposed during pretreatment (Scheme1.a), 73 and removed from the biomass composition as smaller, soluble fragments through the use of a 74 water washing process which produces a black effluent (Scheme1.b1) [18]. To date, the main 75 lignin utilization pathway has been to concentrate the black effluent by evaporation and burn the 76 residual sludgy lignin to supply energy to the pulping plant and biorefinery. Increasingly, 77 however, lignin is being considered as a renewable source of value-added aromatic compounds 78 and fuels $[19,20]$. Significant economic barriers to use of this feedstock arise from the cost of 79 chemical plant equipment needed for various chemical processing steps, and also the cost of 80 chemicals, particularly solvents. Considering the amphiphilic character of lignin (Scheme1.b2), 81 we propose to use the solubilization ability of a commercially available non-ionic, amphiphilic 82 tri-block copolymer, polyethylene oxide- polypropylene oxide- polyethylene oxide (under the 83 trade name of Pluronic ${ }^{\circledR}$ F-68) (Scheme1.c1) toward lignin derivatives to concentrate lignin in 84 the aqueous environment of pulping and pretreatment effluents, greatly reducing costs, and 85 opening possibilities for selective chemistries [21]. Also, five phenolic aromatic model 86 compounds, phenol, eugenol, guaiacol, ferulic acid and guaiacylglycerol-beta-guaiacyl ether 87 (dimer) are employed in order to assist us in studying the solubilization behavior of lignin in 88 micellar system. The uses of block copolymers (i.e., Pluronic ${ }^{\circledR}$ F-68) in solubilizing aromatic 89 and aliphatic molecules in various applications were reported previously; however, the 90 solubilization of lignin in the block copolymer has not yet been studied. 
91 In the present work, we have studied the solubilization behaviors and interactions of lignin and

92 model compounds with Pluronic ${ }^{\circledR}$ F-68 in aqueous ammonia solutions (black effluent) using

93 nuclear magnetic resonance spectroscopy (NMR) and diffusion-ordered nuclear magnetic

94 resonance spectroscopy (DOSY-NMR).

$95 \quad$ Place of Scheme 1

96 Nuclear magnetic resonance spectroscopy (NMR) has been used extensively to characterize the

97 structure, reactions and degradation processes of woody biomass [22]. Most lignin analyses by

98 NMR are done by dissolving lignin from a particular source, treated or untreated, in deuterated

99 dimethyl sulfoxide ( $\mathrm{d}_{6}$-DMSO) and performing a suite of experiments, including $1 \mathrm{D}{ }^{1} \mathrm{H}$ and ${ }^{13} \mathrm{C}$

100 NMR experiments, 2-D correlation experiments, particularly HMQC, HSQC and HMBC, and/or

101 by solid state NMR of extracted and dried lignin samples [23]. Lignin's low solubility in most

102 solvents has been a major hindrance in the structural characterization of lignin polymers.

103 Furthermore, the randomly composed structure of lignin from its three major sub-unit classes (S,

$104 \mathrm{G}$ and $\mathrm{H}$ subunits) provides no regular structure that can be fully characterized by any method.

105 Much effort has been made to refine NMR methods of structural analysis for lignins as a class of

106 compounds because of the great interest in these natural substances by plant biochemists,

107 agronomists, materials scientists, lignin producers, end users for various lignin-derived products,

108 and the sustainable biofuels industry [24]. We have used these methods extensively to investigate

109 lignin structure and pathways of decomposition and reformation for many avenues of lignin

110 modification, including processes of nature [25], e.g. degradation of wheat straw by

111 Phanerochaete chrysosporium [26] and hardwood lignin by the Western Poplar Clearwing Borer,

112 Paranthrene robiniae (Hy. Edwards), [27] as well as lignin digestion in every digestive organ of

113 termites, [28, 29] and more recently, chemical processes, such as heterogeneous catalytic 
114 hydrodeoxygenation of biomass-derived lignin to aromatic hydrocarbons, [30] aqueous ammonia

115 pretreatment of wheat straw, [31] and physical processes including pyrolysis of corn stover [32]

116 and water-only flow-through pretreatment of Miscanthus [33].

117 The knowledge obtained on the solubilization of the lignin in the copolymer micelle will aid to 118 establish processes to convert the lignin into value-added products such as the antioxidant 119 guaiacol, aroma and flavor compound eugenol, or to aromatic hydrocarbons and cycloaliphatics 120 for jet-fuel and provide economics benefit for biorefinery.

\section{2. Materials and methods}

\section{2.1. Materials}

123 PEO-PPO-PEO block copolymer (Pluronic ${ }^{\circledR}$ F-68) was purchased from Sigma-Aldrich 124 (Scheme1-c1). Lignin was obtained from the spent ammonia liquor produced by sequential 125 ozone and soaking aqueous ammonia pretreatment of wheat straw. Phenolic model compounds 126 included phenol (>99\%), guaiacol (98\%), eugenol (>98\%), trans-ferulic acid (99\%), and 127 guaiacylglycerol-beta-guaiacyl ether (dimer) (>97\%) were purchased from Sigma-Aldrich 128 (Milwaukee, USA) and TCI America (Portland Oregon USA), respectively (Fig. 2). Chemicals 129 were used without further purification. NMR sample tubes, $5 \mathrm{~mm} \mathrm{~N} 51 \mathrm{~A} 600 \mathrm{MHz}$, were obtained 130 from Kimble Chase, USA. Solutions for solubilization and $\mathrm{pH}$ adjustment (ammonium 131 hydroxide solution 28-30 \% from Sigma-Aldrich, Milwaukee, USA) were prepared in deuterium 132 oxide $\left(\mathrm{D}_{2} \mathrm{O}, 99.9 \%\right)$ from Cambridge Isotope Laboratories, Inc.

\subsection{Lignin preparation procedure}

134 The spent ammonia liquor produced by the soaking aqueous ammonia pretreatment of wheat 135 straw was recovered through the use of a two step washing process with E-pure water, and the 
136 obtained effluent was gathered for lignin precipitation and purification steps. The pretreatment

137 process has been described in our previous studies [18, 31]. In order to eliminate any interfering

138 effect of non-lignin components in pretreatment effluent on lignin solubilization behavior in the

139 micellar system, a precipitation-purification method disclosed in our previous study [18] was

140 employed with some modifications as needed for application to this study. In general, the

141 precipitation of lignin was performed by adding $20 \mathrm{ml}$ acetic acid : water $(9: 1$, v/v) into effluent

$142(50 \mathrm{ml})$. Then, the precipitated lignin was stored at room temperature $\left(25^{\circ} \mathrm{C}\right)$ for further

143 solubilization study (Scheme1.b1, b2).

144

145

146

147

148

149

150

151

152

153

154

155

156

157

158

\subsection{Critical micelle concentration (CMC) evaluation by DOSY-NMR}

The critical micelle concentration (CMC) of the PEO-PPO-PEO block copolymer (Pluronic ${ }^{\circledR}$ F68) was evaluated by diffusion ordered nuclear magnetic resonance spectroscopy (DOSY-NMR).

The micelle copolymer solutions were prepared by direct dissolution of the copolymer in $\mathrm{D}_{2} \mathrm{O}$ at different concentrations (mM), and values for the diffusion coefficients $D$ were determined for each concentration. The CMC was determined from the inflection point of a plot of diffusion coefficient vs. polymer concentration. DOSY-NMR is a two dimensional NMR technique, usually employing a magnetic field gradient along the z-axis of the sample, in which the NMR signal decays exponentially with increasing gradient power, according to the diffusion behavior of individual molecules. This leads to two dimensions; the first dimension $(F 2)$ accounts for the conventional chemical shift and the second one $(F 1)$ for diffusion coefficient $(D)$ [34]. DOSY NMR measurements were performed on a Varian VNMRS 600 (liquid) NMR spectrometer operating at $600 \mathrm{MHz}$, equipped with z-axis pulsed field gradients. A Varian pulse sequence DgcsteSL_cc (gradient compensated stimulated echo with spin lock and convection compensation) was employed, incorporating both convection compensation and eddy current 
159

160

161

162

163

164

165

166

167

168

169

170

171

172

173

174

175

176

177

178

179

180

compensation. Samples were temperature equilibrated at $25^{\circ} \mathrm{C}$ for at least 20 minutes prior to acquisition. An array of 15 gradient power level settings was used with a fixed gradient length of 5ms and diffusion delay of 50ms. Sweep width, acquisition time and PRESAT parameters were the same as for the 1D experiments, except that a purge cycle for water suppression was excluded to avoid RF heating of the sample, and the relaxation delay was decreased to 5 s. 8192 complex data points were collected. Processing of the data involved application of line broadening $(2 \mathrm{~Hz})$ prior to Fourier transform and baseline correction, followed by a multicomponent fit (number of components $=4-6$ ) of the arrayed frequency spectra to a modified Stejskal-Tanner equation (Eq. 1) employing a polynomial correction factor for non-uniform gradients.

$S(G)=S(0) \exp \left[-\Sigma_{n=1}^{5}\right.$ nugcal $\left.n \eta^{n} G^{2 n}\right]$

Where, $\eta=D \gamma^{2} \delta^{2} \Delta$ and $\mathrm{G}$ is the nominal gradient amplitude.

\subsection{Solubilization study by liquid-state ${ }^{1} H$ NMR}

A Stock solution of the PEO-PPO-PEO tri-block copolymer (Pluronic ${ }^{\circledR}$ F-68) was prepared by dissolving the appropriate amount of the block copolymer in deuterium oxide (99\%) under gentle agitation, such that the resulting stock solution $(50 \mathrm{ml})$ had a concentration of $0.04 \mathrm{mM}$ (at the CMC). To make the concentration series for each model compound (guest), known volumetric amounts of the solute were added to aliquots of stock solution $(1 \mathrm{ml})$ of the tri-block copolymer. Lignin was weighed as a solid to make weight per volume dilutions. This resulted in preparation of six samples with different concentrations $(0.01,0.04,0.08,0.12,0.16,0.2 \mathrm{mM})$ for each tested material. The $\mathrm{pH}$ of all samples was adjusted to 11.4 using ammonium hydroxide. Preparation of solutions and the solubilization process took place at room temperature $\left(25^{\circ} \mathrm{C}\right)$ without heating 
181 or mechanical stirring. The solutions were then stored for 24 hour to equilibrate before

182

183

184

185

186

187

188

189

190

191

192

193

194

195

196

197

198

199

200

201

202

203

assessment of solubilization by NMR. $700 \mu \mathrm{l}$ of each sample solution was injected into a $5 \mathrm{~mm}$ NMR sample tube with a syringe, and the NMR tube was sealed with a plastic tube cap.

${ }^{1} \mathrm{H}$ NMR spectra were obtained at $25^{\circ} \mathrm{C}$ in $\mathrm{D}_{2} \mathrm{O}$ (Isotech, 99.8\%D) solution using a Varian/Agilent 600MHz NMR spectrometer equipped with a DD2 console and One Probe multinuclear probe and z-axis pulsed field gradients. Water suppression (PRESAT/purge, 2-step) was used due to residual water arising from use of aqueous ammonium hydroxide to adjust $\mathrm{pH}$ to 11.4. All spectra were taken using a calibrated 90 degree pulse and relaxation delay of 15 seconds. A minimum of 16 scans were required at the lowest concentrations of model compounds, and up to 128 scans were collected in some cases to reach acceptable signal-to-noise ratios. Throughout the study a sweep width of $6313.1 \mathrm{~Hz}$ was used, acquisition time 1.3 seconds, and 16384 data points. ${ }^{13} \mathrm{C}$-decoupling was not employed. Line broadening of $1.5 \mathrm{~Hz}$ was used prior to Fourier transform of each data set. All resonances were referenced to the residual water peak at $4.65 \mathrm{ppm}$. Chemical shifts resulting from interactions of the A-B-A-block-co-polymer with model compounds and lignin were measured in hertz relative to the first concentration data point. In most cases, a blank polymer spectrum (no added model compound) was not recorded.

\section{Result and discussion}

Although our end goal is to show that hydrophilic and aromatic interactions between lignin and block copolymer can form a type of micelle structure in which diverse reaction chemistry, some previously unavailable in aqueous solution, can be performed efficiently on lignin to produce value-added products, we decided that it was of paramount importance to first study the formation of micelles in our proposed system, as well as their ability to solubilize guest molecules, including partially decomposed lignin from process streams, and to elucidate the 
204 interactions that occur between host micelles and guest model compounds representing lignin 205 structural features, and lignin itself.

\section{3.1. Critical micelle concentration $(C M C)$ determination}

207 Amphiphilic molecules spontaneously self-assemble, at or above a critical concentration in 208 aqueous solutions, to form aggregates of various morphologies $[35,36]$. The resulting micelles 209 are stabilized in water by the favorable interactions between hydrophilic groups and water 210 molecules, forming a stable hydrated shell between the hydrophobic core and the bulk water. For 211 ionic surfactants, the shell surrounding the core is a charged layer, or stern layer, while for non-

212 ionic surfactants it is referred to as a hydrated palisade layer [37]. Techniques which monitor 213 formation of micelles, rather than changes in aggregation number, are preferable for the CMC 214 determination of block copolymer micelles [38]. Many techniques have been used to determine 215 CMC values, including fluorescence [39], light scattering [40, 41], osmometry [42], viscosity 216 [43] and DOSY NMR [44, 45]. In this study, the CMC of the PEO-PPO-PEO block copolymer 217 (Pluronic ${ }^{\circledR}$ F68) solution in deuterium oxide was estimated by diffusion ordered nuclear 218 magnetic resonance spectroscopy (DOSY-NMR). A plot of concentration of polymer vs. 219 diffusion coefficient (D) reveals an inflection at $0.04 \mathrm{mM}(\mathrm{CMC}=0.04 \mathrm{mM})$ at both $\mathrm{pH} 7.5$ and 220 11.4. This demonstrates that the critical micelle concentration of this polymer in aqueous 221 solution is not altered at high $\mathrm{pH}$.

222 A further study of the effect of added model compounds on the critical micelle concentration of 223 the block copolymer was carried out by DOSY NMR. Diffusion coefficients of copolymer 224 solutions at $\mathrm{pH}$ 11.4-after addition of the model compounds-were obtained at the CMC of the 225 polymer $(0.04 \mathrm{mM})$. As shown in Figure 1, the CMC was not significantly altered by addition of 226 selected guest model compounds at a guest concentration of $0.04 \mathrm{mM}$. At a three-fold higher 
227 loading of guests $(0.12 \mathrm{mM})$, however, the diffusion coefficient of the Pluronic F-68 PEO block

228 generally decreased with the addition of these model compounds, consistent with additional

229 loading of guests and/or reordering of the micelle structure. Based on the above results, a 230 concentration of $0.04 \mathrm{mM}$ Pluronic ${ }^{\circledR}$ F-68 was used throughout the solubilization study.

231 Place of Fig.1.

232 3.2. Solubilization of solute in micellar aqueous solution: Effect of $\pi-\pi$ aromatic stacking and 233 hydrophilic interactions on the micelle-lignin structure

234 NMR studies of solubilization of model phenolic compounds and lignin in PEO-PPO-PEO block 235 copolymer micelles revealed that all the models tested in this study were able to solubilize into 236 these micelles, presumably due to the hydrophobic interaction between the core of the micelles 237 and aromatic rings of the solute. As seen in Fig. 2 (a-b), Group A model compounds primarily 238 interact with the oxyethylene blocks (polyethylene oxide) of the copolymer, compared to Group 239 B model compounds which mainly affected the polypropylene mid-block of the copolymer. 240 Patterns of solubilization-interaction behavior of model phenolic compounds (Groups A and B) 241 and partially decomposed lignin with copolymer micelles was investigated by monitoring ${ }^{1} \mathrm{H}$ 242 NMR chemical shift changes for PEO- $\mathrm{CH}_{2}-\mathrm{CH}_{2}$ resonances, and for methyl resonances of the B243 block (micellar core, PPO- $\mathrm{CH}_{3}$ ) of Pluronic ${ }^{\circledR}$ F-68 block copolymer as a function of 244 concentration of the tested model compounds, similar to the method described by Kim, Im and 245 Oh for sodium dodecylsulfate micelles [46].

$246 \quad$ Place of Figs.2. $(a \& b)$

247 3.2.1. Solubilization of model phenolic compounds phenol, guaiacol, eugenol (group A) in PEOPPO-PEO block copolymer micelles 
249 As can be seen in Fig. 2(a), at low model concentrations there is a gradual upfield shift in the

250 PEO- $\mathrm{CH}_{2}-\mathrm{CH}_{2}$ resonances with increasing concentration of model, which is mirrored by a

251 chemical shift change in the $\mathrm{PPO}-\mathrm{CH}_{3}$ resonances. The small, almost linear change in chemical

252 shifts proceeds until a "critical guest concentration" (CGC) is reached, whereupon a large

253 chemical shift is observed, indicating a reordering of the structure of the micelles. The greatest

254 chemical shifts observed at a guest concentration of $0.20 \mathrm{mM}$ of each, eugenol, phenol, and 255 guaiacol were, for eugenol, $282.5 \mathrm{~Hz}$ for $\mathrm{PEO}-\mathrm{CH}_{2}-\mathrm{CH}_{2}$ resonances and $195.2 \mathrm{~Hz}$ for $\mathrm{PPO}-\mathrm{CH}_{3}$ 256 resonances of the polymer; for phenol $233.4 \mathrm{~Hz}$ and $150 \mathrm{~Hz}$, respectively; and for guaiacol $257273.3 \mathrm{~Hz}$ and $173.6 \mathrm{~Hz}$, respectively. The changes in chemical shift are concentration dependent 258 and are non-uniform, particularly at the concentrations $0.04 \mathrm{mM}$ in eugenol, $0.12 \mathrm{mM}$ in phenol 259 and $0.16 \mathrm{mM}$ in guaiacol, where an inflection occurs. The dramatic chemical shift changes are 260 accompanied by clouding of the solution, as a macroscopic emulsion forms (Fig. 3, photos). 261 These emulsions remain stable in solution for weeks, and do not further aggregate or release their 262 payloads. The upper limit of micellar loading for each model compound has not yet been 263 determined. The graphs in Fig. 2(a) clearly show that above the CGC, the chemical shift 264 differences $(\Delta \delta)$ of polymer $\mathrm{CH}_{3}$ resonances versus $\mathrm{CH}_{2}-\mathrm{CH}_{2}$ resonances differ by as much as $265100 \mathrm{~Hz}$, indicating a differential effect of the guest on polymer PEO regions over PPO regions. In 266 general, for group A model compounds, proton chemical shift changes $(\Delta \delta)$ for PEO methylene 267 resonances (palisade region of micelles) was greater than for the PPO methyl (core) resonances. 268 The greater magnitude of chemical shift change for the PEO block indicates that the guest is 269 preferentially oriented within the hydrated palisade layer of the micelle, but also extends at least 270 partially into the core. 
271 Further insight into the morphological transitions of micelles incorporating aromatic solute can

272 be gleaned from the literature. In nonionic PEO-PPO-PEO block copolymer micelles, the

273 hydrophilic palisade layer comprising the hydrated PEO blocks controls the structural properties

274 of the micelles (micellar shape), maintaining a hydrophobic core and hydrophilic shell in

275 aqueous solution [38]. It has been stated that dehydration of the PEO shell causes a type of shape

276 change in micelles [48, 49]. Kadam [47] described the effect of added salt on Tetronic T904

277 aggregation behavior in aqueous solution. The authors reported that the presence of salt

278 dehydrates the PEO shell, and consequently the spherical shape of the micelles is changed to

279 prolate ellipsoidal. The influence of salicylic acid on the aggregation characteristics of Pluronic ${ }^{\circledR}$

280 P85 has been studied in the aqueous medium by Parekh and coworkers [48]. The authors stated

281 that salicylic acid resides in the micellar shell and consequently dehydrates the corona region,

282 resulting in a sphere-to-rod shape transition. Dehydration of core and shell regions of micelles is

283 associated with restructuring and growth processes of micelles. Dey and coworkers [49] reported

284 that corona specific micellar dehydration by $\mathrm{NaCl}$ induces inter-micellar interaction and 285 consequent formation of micellar clusters. On the other hand, it also was stated that core specific 286 micellar dehydration by butan-1-ol brings about sphere-to-rod micellar shape transition on 287 approaching the cloud point of copolymer solutions. Similarly, in the model compounds Group 288 A, the structural transitions made apparent in both $\mathrm{PEO}-\mathrm{CH}_{2}-\mathrm{CH}_{2}$ and $\mathrm{PPO}-\mathrm{CH}_{3}$ regions of the 289 NMR spectrum (as well as in the aromatic signals for the solute) were accompanied with 290 clouding of the samples (Fig. 3, photos). It was found that the phenolic model compounds, 291 phenol, guaiacol and eugenol predominantly affected the palisade layer of the micelles, and 292 PEO- $\mathrm{CH}_{2}-\mathrm{CH}_{2}$ resonances shifted upfield significantly (Fig. 2a). The observed $\pi-\pi$ aromatic 293 interaction of Group A model compounds is indicated in the aromatic region of the NMR 
294 spectrum as well, especially in eugenol and guaiacol, as individual guest molecules stack in an 295 alternating orientation, such that methoxy groups occupy an opposite configuration on each 296 alternating stack member, relieving steric interactions between the methoxys. The reordering of 297 solute in the micelles by $\pi-\pi$ interactions may allow more efficient loading of guest within 298 micelle, and could also result in the dehydration of the palisade region and a gross structural 299 transition in the micelle.

300 The changes in morphology of the micelles for Group A model compounds is thought to arise 301 from $\pi-\pi$ interactions between guest molecules, which have the opportunity to achieve increased 302 order or stacking within the scaffold provided by the polymer chains of the A-B-A block 303 copolymer micellar structure. In contrast, $\pi-\pi$ interactions of model compounds in free solution is 304 not observable by NMR. For a given model compound, it is apparent that the buildup of $\pi-\pi$ 305 interactions is dependent on the chemical structure of a given model, such that eugenol has the 306 lowest CGC $(\sim 0.05 \mathrm{mM})$, followed by phenol, then guaiacol. It is apparent that the methoxy 307 group plays a role in promoting $\pi-\pi$ interactions in these systems, but it is also clear that more 308 than one effect determines the extent and form of $\pi-\pi$ interactions, the CGC for a given model, 309 and the loci of the models within their host micelles. Future studies will expand the concentration 310 range studied and the diversity of lignin model compounds, and will attempt to determine the 311 shape and size of the micelles obtained.

312 3.2.2. Soubilization of model phenolic compounds ferulic acid, guaiacylglycerol-beta-guaiacyl 313 ether, and partially decomposed lignin (Group B) in PEO-PPO-PEO block copolymer micelles

314 Patterns of solubilization behavior for ferulic acid, lignin, and guaiacylglycerol-beta-guaiacyl 315 ether in the micellar system are shown in Fig. 2(b). For Group B models, the magnitude of $\Delta \delta$ is 
much smaller, in general, than for Group A. The greatest chemical shifts observed for ferulic acid were $45.2 \mathrm{~Hz}$ for $\mathrm{PEO}-\mathrm{CH}_{2}-\mathrm{CH}_{2}$ resonances and $46.9 \mathrm{~Hz}$ for $\mathrm{PPO}-\mathrm{CH}_{3}$; for lignin, $35.5 \mathrm{~Hz}$ and $13.82 \mathrm{~Hz}$ for $\mathrm{PPO}-\mathrm{CH}_{3}$ and $\mathrm{PEO}-\mathrm{CH}_{2}-\mathrm{CH}_{2}$ signals, respectively; and for guaiacylglycerolbeta-guaiacyl ether were only $6.94 \mathrm{~Hz}$ for both $\mathrm{PEO}-\mathrm{CH}_{2}-\mathrm{CH}_{2}$ and $\mathrm{PPO}-\mathrm{CH}_{3}$ resonances. After addition of Group B model compounds to the block copolymer solution, the magnitude of chemical shifts of both PPO methyl protons and PEO oxyethylene protons followed the order of ferulic acid > lignin > guaiacylglycerol-beta-guaiacyl ether. Having the highest rate of chemical shift change at lower concentrations, ferulic acid stands out, and can be compared qualitatively with eugenol, in that both models have a propenyl side chain, but ferulic acid has a carboxylic acid group at the terminus of its propyl chain. Ferulic acid shows an earlier onset of interaction with polymer, but addition of eugenol results in a much greater chemical shift effect, and greater differentiation between core and shell regions of the micelle, as evidenced by the difference in magnitude of chemical shift change between $\mathrm{PEO}-\mathrm{CH}_{2}-\mathrm{CH}_{2}$ vs. $\mathrm{PPO}-\mathrm{CH}_{3}$ for eugenol. In the case of ferulic acid, PEO methylene and PPO methyl signals move upfield at virtually the same rate, and level off together, suggesting there is little differentiation between core and shell in the distribution of ferulic acid in the micelle. Thus, we conclude that monolignol-like model compounds having polar, hydrogen bonding substituents interact with both core and shell regions in micelles of PEO-PPO-PEO block co-polymers roughly equally.

There is a third factor at work in the reordering of micelles incorporating guest molecules modeled on lignin. The polyoxyethylene chains of PEO-PPO-PEO block copolymers that cover the micelle core are hydrated. It has been reported that each oxyethylene unit binds around three water molecules (in the unloaded polymer), and that the bound water is relatively equally distributed through the head group layer [50]. It has also been shown that the water solubility of 
339 polymer aggregates increases with the presence of polar substituents on solute, and this helps the

340 compound dissolve not only in the most hydrophobic part of the micelle but also in the less

341 hydrophobic palisade region [51]. The same observation was reported by Tehrani-Bagha and

342 Holmberg [52] that the dyes, acid orange 20 and basic yellow 2, were able to dissolve in the

343 palisade layer of the nonionic surfactant. The polarity gradient present between hydrophilic shell

344 and hydrophobic core regions of the micelle provides that both nonpolar and polar reagents-or

345 amphiphiles-can be solubilized with preferential orientation within the micelle [3, 4]. This highly

346 ordered solubilization results in selective effects [21], which lend themselves well to micellar

347 catalysis and acceleration of the rate of reactions [53, 54], a primary goal of our research group.

348 One effect of polar groups is to dampen the effect of $\pi-\pi$ interactions within the micelles. The 349 carboxylic acid moiety of ferulic acid, with respect to eugenol, causes an apparent drastic 350 reduction in chemical shift due to interactions with polymer chains, and causes the relative $\Delta \delta$ 351 between PEO and PPO resonances to greatly decrease in magnitude, and virtually eliminates the 352 differentiation of ferulic acid between core and shell regions. The existence of $\pi$ - $\pi$ interactions in 353 this system is by no means ruled out, but the effect of hydrogen bonding from the carboxylic acid 354 seems to dominate the overall interaction. There is no bulk transition in morphology of micelles 355 in the case of ferulic acid (and no clouding observed). Interestingly, for the guaiacylglycerol356 beta-guaiacyl ether (dimer), the effect of primary and benzylic alcohol functions on the propyl 357 bridge of the guaiacylglycerol-beta-guaiacyl ether is to shunt the magnitude of polymer-model 358 interactions further, at least at the concentrations studied. The guaiacylglycerol-beta-guaiacyl 359 ether, in fact, follows the same pattern observed for guaiacol throughout the concentration range 360 studied. Whereas guaiacol shows a late transition in reordering of micellar structure, 361 accompanied by differentiation between core and shell regions, the guaiacylglycerol-beta- 
362 guaiacyl ether shows only a small, incremental chemical shift progression, with no significant 363 differentiation at the concentrations studied. However, the guaiacylglycerol-beta-guaiacyl ether 364 did show tangible evidence of $\pi-\pi$ interactions, based on the coalescence of chemical shifts in the 365 aromatic region. This is further evidence that both $\pi-\pi$ interactions and hydrogen bonding play 366 important roles in the solubilization behavior of lignin in these micellar systems.

367 Partially decomposed lignin showed the most interesting and unexpected results in this 368 concentration study of Group B model compounds. Having many possible distributions of S, G 369 and $\mathrm{H}$ monolignol units, lignin has a full range of possible electronic and steric effects that can 370 influence the electronic environment of polymer chains, and their corresponding NMR signals, 371 upon incorporation into the micelle. One might expect the solution structure of lignin to be 372 homogeneous, such that all of its possible groups and molecular organizations exist randomly 373 and equally in solution. It can be seen from Fig. 2(b), however, that there is a differentiation of 374 lignin between core and shell which is immediate, and which increases with concentration. Even 375 more striking is that this differentiation of lignin between core and shell regions is opposite all of 376 the small molecule models studied in which $\pi-\pi$ interactions and/or bulk reorganizations were 377 observed, in that lignin interactions with core PPO methyls dominate over PEO methylenes in 378 the shell. There is a discernible inflection of both PPO and PEO curves at around 0.14mM lignin, 379 which results in a dramatically greater $\Delta \delta$ with respect to earlier concentrations, leading to the 380 conclusion that there is at least a moderate $\pi-\pi$ interaction effect with increasing lignin 381 concentration in the micelle, and possibly some change in morphology of the micelles 382 themselves. However, no clouding was observed in the aqueous sample at the upper 383 concentrations. We are actively seeking to determine the size and morphology of the polymer 
384 micelles, and further elaborate any changes in morphology with increasing concentration of 385 lignin.

386 The chemical shift difference between polymer PEO block methylenes and PPO methyls is 387 immediately apparent at low concentrations of solubilized lignin in the micelles, and remains 388 relatively constant $(\Delta \delta=\sim 7-8 \mathrm{~Hz})$ up to $0.12 \mathrm{mM}$ lignin. It has been a longstanding belief that 389 partially decomposed lignin in certain pulp process effluent solutions has some inherent and 390 ordered aggregation due to $\pi-\pi$ interactions. Aggregation of solubilized lignin was first observed 391 by Benko in 1964 [55]. Two dominant views of the driving force behind lignin aggregation have 392 been proposed. Lindstrom [56] described the role of hydrogen bonding between carboxylic acid 393 groups of lignin and hydroxyls and other oxygen functions in lignin structures, while Sarkanen 394 and Glasser $[57,58]$ proposed that $\pi-\pi$ interactions between aromatic rings in lignin were 395 responsible for the aggregation phenomenon, and showed that acetylation of soluble lignin had 396 no effect on aggregation, thus diminishing the role of hydrogen bonding interactions. The 397 inherent ordering of lignin in solution due to " $\pi$-stacking" has recently been more thoroughly 398 studied by Deng [59] who demonstrated that the $\pi-\pi$ interactions could be disrupted by formation 399 of charge-transfer complexes between iodine and the stacked aromatic rings, which resulted in 400 dis-aggregation of lignin aggregates.

$401 \quad$ Place of Fig.3.

402 The current study sheds some light on that argument. At the inflection point (or CGC) of the 403 curve for lignin in Fig. 2(b) the difference in $\Delta \delta$ between PEO methylenes and PPO methyls 404 roughly triples, indicating a reordering of the guest model-host polymer system. This is a weaker 

$421 \pi$ interactions and hydrogen bonding.

422 Given these results, we infer that two primary driving forces govern the solubilization behavior 423 and structural properties of micelle-solute assemblies: (1) the interaction of oxygenated

424 functional groups of lignin, especially in the propanoid chain, with polyoxyethylene A-blocks of 425 the copolymer, and (2) $\pi-\pi$ interactions between lignin (or model) aromatic rings. We also infer 426

transition than for Group A model compounds, however, we postulate that the observed chemical shift data represents the net effect of two competing forces, hydrophilic and $\pi-\pi$ interactions.

\section{Conclusion}

In this work, ${ }^{1} \mathrm{H}$ NMR diffusion ordered spectroscopy (DOSY) was used to confirm the critical micelle concentration for a selected, commercially available ABA block copolymer (trade name Pluronic $^{\circledR}$ F-68) at neutral and high pH. Results show that the incorporation of guest molecules did not disrupt the integrity of these micelles. Using ${ }^{1} \mathrm{H}$ NMR spectroscopy, we found that increasing the concentration of guaiacol, eugenol, or phenol (Group A model compounds) resulted in a type of macroscopic restructuring of the micelle. A dramatic upfield chemical shift of PEO- $\left(\mathrm{CH}_{2}-\mathrm{CH}_{2}\right)$ - and PPO- $\left(\mathrm{CH}_{3}\right)$ proton resonances occurred at a critical guest concentration (CGC), accompanied by gross structural transitions in the micelle, clouding of the solution, and formation of highly stable macroscopic emulsions. Although lignin and the model compounds ferulic acid and guaiacylglycerol- $\beta$-guaiacyl ether (Group B model compounds) also showed concentration-dependent chemical shifts, large structural transitions seen in the Group A models did not produce visible changes in micellar solutions of Group B compounds. Nonetheless, liquid state ${ }^{1} \mathrm{H}$ NMR revealed complex behavior for these model-polymer interactions driven by both $\pi$ -

that the hydration/dehydration of the palisade layer likely plays a role in the structural transitions 
427 of micelle-solute structures, and is controlled by the relative levels of polar, hydrogen bonding

428 substituents in a particular guest. Changes in the microscopic structure of the palisade layer due

429 to guest incorporation are probably balanced by changes in hydration of the oxyethylene chains

430 in the palisade region of the micelle, which can easily be hydrated or dehydrated in response to

431 the influence of a particular guest or solute. We conclude that the overall effect for partially

432 decomposed lignin from process effluent streams is solubilization of lignin with a preferred

433 orientation in the micelle, guided by these distinct influences.

\section{Acknowledgment}

435 This material is based upon work supported by the National Science Foundation under Grant No. 4361231085 and Washington State University. The authors would like to acknowledge the Center 437 for NMR spectroscopy at WSU. The WSU NMR Center equipment was supported by NIH 438 grants RR0631401 and RR12948, NSF grants CHE-9115282 and DBI-9604689, the Murdock 439 Charitable Trust, and a generous donation by Don and Marianna Matteson. Also, the authors 440 would like to acknowledge Jonathan Lomber, Analytical Lab Manager, of the Department of 441 Biological Systems Engineering, Washington State University.

\section{Corresponding authors}

443 LJ Smith Hall 213, Department of Biological Systems Engineering, Washington State University,

444 Pullman, WA, 99164-6120, USA.

445 *Tel.: +1 408805 2057; E-mail address: mohammadali.azadfar@wsu.edu (M. Azadfar)

446 ** Tel.: +1 509335 3743; E-mail address: chens@wsu.edu (S.Chen)

447 Appendix A. Supplementary data

448 Supplementary data associated with this article can be found, in the online version, at 


\section{References}

[1] Nagarajan, R., Ganesh, K. Block copolymer self-assembly in selective solvents: theory of solubilization in spherical micelles. Macromolecules. 11 (1989) 4312-4325.

[2] Goyal, P.S., Aswal, V.K. Micellar structure and inter-micelle interactions in micellar solutions: results of small angle neutron scattering studies. Current Science-Bangalore. 8 (2001) 972-979.

[3] Dwars, T., Paetzold, E., Oehme, G. Reactions in micellar systems. Angewandte Chemie International Edition. 44 (2005) 7174-7199.

[4] Tehrani-Bagha, A.R., Holmberg, K. Solubilization of hydrophobic dyes in surfactant solutions. Materials. 2 (2013) 580-608.

[5] Xing, L., Mattice, W.L. Strong solubilization of small molecules by triblock-copolymer micelles in selective solvents. Macromolecules. 6 (1997) 1711-1717.

[6] Webber, S.E. Polymer micelles: an example of self-assembling polymers. The Journal of Physical Chemistry B. 15 (1998) 2618-2626.

[7] Nagarajan, R., Barry, M., Ruckenstein, E. Unusual selectivity in solubilization by block copolymer micelles. Langmuir. 2 (1986) 210-215.

[8] Quintana, J.R., Salazar, R.A., Katime, I. Solubilization of Homopolymers by Block Copolymer Micelles in Dilute Solutions: Laser Light Scattering and Viscosity Studies on Micellar Solutions. The Journal of Physical Chemistry. 11 (1995) 3723-3731.

[9] Xu, W., Ling, P., Zhang, T. Polymeric micelles, a promising drug delivery system to enhance bioavailability of poorly water-soluble drugs. Journal of drug delivery. (2013) 1-15.

[10] Shi, H., Qi, L., Ma, J., Cheng, H. Polymer-directed synthesis of penniform BaWO4 nanostructures in reverse micelles. Journal of the American Chemical Society. 12 (2003) 34503451.

[11] Rathman, J.F. Micellar catalysis. Current Opinion in Colloid \& Interface Science. 4 (1996) 514-518.

[12] Hurter, P.N., Hatton, T.A. Solubilization of polycyclic aromatic hydrocarbons by poly (ethylene oxide-propylene oxide) block copolymer micelles: effects of polymer structure. Langmuir. 5 (1992) 1291-1299.

[13] Gadelle, F., Koros, W.J., Schechter, R.S. Solubilization of aromatic solutes in block copolymers. Macromolecules. 14 (1995) 4883-4892.

[14] Heins, A., McPhail, D.B., Sokolowski, T., Stöckmann, H., Schwarz, K. The location of phenolic antioxidants and radicals at interfaces determines their activity. Lipids. 46 (2007) 573582. 
[15] Kumar, S., David, S.L. Effects of various hydrocarbons on micellar growth. Journal of the American Oil Chemists' Society. 77 (1997) 797-801.

[16] Farrell, R.A., Fitzgerald, T.G., Borah, D., Holmes, J.D., Morris, M.A. Chemical interactions and their role in the microphase separation of block copolymer thin films. International journal of molecular sciences. 9 (2009) 3671-3712.

[17] Buranov, A.U., Mazza, G. Lignin in straw of herbaceous crops. Industrial crops and products. 3 (2008) 237-259.

[18] Azadfar, M., Gao, A.H., Bule, M., Chen, S. Structural characterization of lignin: a potential source of antioxidants guaiacol and 4-vinylguaiacol. International journal of biological macromolecules. 75 (2015) 58-66.

[19] Helander, M., Theliander, H., Lawoko, M., Henriksson, G., Zhang, L., Lindström, M.E. Fractionation of technical lignin: Molecular mass and pH effects. BioResources. 2 (2013) 22702282.

[20] Hamaguchi, M., Cardoso, M., Vakkilainen, E. Alternative technologies for biofuels production in kraft pulp mills_-Potential and prospects. Energies. 7 (2012) 2288-2309.

[21] Hurter, P.N., Scheutjens, J.M., Hatton, T.A. Molecular modeling of micelle formation and solubilization in block copolymer micelles. 1. A self-consistent mean-field lattice theory. Macromolecules. 21 (1993) 5592-5601.

[22] Maunu, S.L. NMR studies of wood and wood products. Progress in Nuclear Magnetic Resonance Spectroscopy. 2 (2002) 151-174.

[23] Laskar, D.D., Jourdes, M., Patten, A.M., Helms, G.L., Davin, L.B., Lewis, N.G. The Arabidopsis cinnamoyl CoA reductase irx4 mutant has a delayed but coherent (normal) program of lignification. The Plant Journal. 5 (2006) 674-686.

[24] Gao, X., Laskar, D.D., Zeng, J., Helms, G.L., Chen, S. A 13C CP/MAS-Based Nondegradative Method for Lignin Content Analysis. ACS Sustainable Chemistry \& Engineering. 1 (2014) 153-162.

[25] Dey Laskar, D., Ke, J., Zeng, J., Gao, X., Chen, S. Py-GC/MS as a powerful and rapid tool for determining lignin compositional and structural changes in biological processes. Current Analytical Chemistry. 3 (2013) 335-351.

[26] Singh, D., Zeng, J., Laskar, D.D., Deobald, L., Hiscox, W.C., Chen, S. Investigation of wheat straw biodegradation by Phanerochaete chrysosporium. Biomass and bioenergy. 33 (2011) 1030-1040.

[27] Ke, J., Laskar, D.D., Chen, S. Biodegradation of hardwood lignocellulosics by the western poplar clearwing borer, Paranthrene robiniae (Hy. Edwards). Biomacromolecules. 15 (2011) 1610-1620. 
521 [28] Ke, J., Laskar, D.D., Singh, D., Chen, S. In situ lignocellulosic unlocking mechanism for 522 carbohydrate hydrolysis in termites: crucial lignin modification. Biotechnol. Biofuels. 1 (2011) 52317.

524 [29] Ke, J., Laskar, D.D., Chen, S. Varied lignin disruption mechanisms for different biomass 525 substrates in lower termite. Renewable Energy. 50 (2013) 1060-1064.

526 [30] Laskar, D.D., Tucker, M.P., Chen, X., Helms, G.L., Yang, B. Noble-metal catalyzed 527 hydrodeoxygenation of biomass-derived lignin to aromatic hydrocarbons. Green Chemistry. 2 528 (2014) 897-910.

529

530

531

532

533

534

535

[31] Gao, A.H., Bule, M.V., Laskar, D.D., Chen, S. Structural and thermal characterization of wheat straw pretreated with aqueous ammonia soaking. Journal of agricultural and food chemistry. 35 (2012) 8632-8639.

[32] Yang, X., Laskar, D.D., Ma, F., Zhang, X., Chen, S. Medium-Temperature Pyrolysis of Corn Stover Improved by Biopretreatment with White-rot Fungi. BioResources. 4 (2013) 63836394.

[33] Laskar, D.D., Zeng, J., Yan, L., Chen, S., Yang, B. Characterization of lignin derived from water-only flowthrough pretreatment of Miscanthus. Industrial Crops and Products. 50 (2013) 391-399.

[34] Bakkour, Y., Darcos, V., Li, S., Coudane, J. Diffusion ordered spectroscopy (DOSY) as a powerful tool for amphiphilic block copolymer characterization and for critical micelle concentration (CMC) determination. Polymer Chemistry. 8 (2012) 2006-2010.

[35] Lindman, B., Wennerström, H. Micelles. Springer Berlin Heidelberg. 78 (1980) 1-83.

[36] Zana, Raoul, ed. Dynamics of surfactant self-assemblies: micelles, microemulsions, vesicles and lyotropic phases. CRC press. 125 (2005) 1-536.

[37] Evans, D.F., Wennerstrom, H. Colloidal domain. Wiley-VCH. 2(1999) 1-632.

[38] Gao, Z., Eisenberg, A. A model of micellization for block copolymers in solutions. Macromolecules. 26 (1993) 7353-7360.

[39] Astafieva, I., Zhong, X.F., Eisenberg, A. Critical micellization phenomena in block polyelectrolyte solutions. Macromolecules. 26 (1993) 7339-7352.

[40] Quintana, J.R., Villacampa, M., Katime, I.A. Micellization of a polystyrene-b-poly (ethylene/propylene) block copolymer in n-dodecane/1, 4-dioxane mixtures. 2. Structure and dimensions of micelles. Macromolecules. 4 (1993) 606-611.

[41] Tuzar, Z., Konak, C., Stepanek, P., Plestil, J., Kratochvil, P., Prochazka, K. Dilute and semidilute solutions of ABA block copolymer in solvents selective for A or B blocks: 2. Light scattering and sedimentation study. Polymer. 11 (1990) 2118-2124.

[42] Price, C. Micelle formation by block copolymers in organic solvents. Pure and Applied Chemistry. 10 (1983) 1563-1572. 
557 [43] Sikora, A., Tuzar, Z. Association of two-block copolymer polystyrene-block-poly 558 (2-vinylpyridine) in toluene. Die Makromolekulare Chemie. 10 (1983) 2049-2059.

559 [44] Zawaneh, P.N., Doody, A.M., Zelikin, A.N., Putnam, D. Diblock copolymers based on 560 dihydroxyacetone and ethylene glycol: Synthesis, characterization, and nanoparticle 561 formulation. Biomacromolecules. 11 (2006) 3245-3251.

562 [45] Ahn, S., Kim, E.H., Lee, C. Diffusion-Ordered NMR Spectroscopy of Poly ([ethylene-co563 vinyl acetate]-graft-vinyl chloride) in Solution. Bulletin of the Korean Chemical Society. 2 564 (2005) 331-333.

565 [46] Kim, B.J., Im, S.S., Oh, S.G. Investigation on the solubilization locus of aniline-HCl salt in 566 SDS micelles with 1H NMR Spectroscopy. Langmuir. 2 (2001) 565-566.

567 [47] Kadam, Y., Singh, K., Marangoni, D.G., Ma, J.H., Aswal, V.K., Bahadur, P. Induced 568 micellization and micellar transitions in aqueous solutions of non-linear block copolymer 569 Tetronic ${ }^{\circledR}$ T904. Journal of colloid and interface science. 2 (2010) 449-456.

570 [48] Parekh, P. Room temperature sphere-to-rod growth of Pluronic ${ }^{\circ}$ P85 micelles induced by 571 salicylic acid. Soft Matter 8.21 (2012) 5864-5872.

572 [49] Dey, J., Kumar, S., Nath, S., Ganguly, R., Aswal, V.K., Ismail, K. Additive induced core 573 and corona specific dehydration and ensuing growth and interaction of Pluronic F127 574 micelles. Journal of colloid and interface science. 415 (2014) 95-102.

575 [50] Carlström, G., Halle, B. The state of water in non-ionic surfactant solutions and lyotropic 576 phases. Oxygen-17 magnetic relaxation study. Journal of the Chemical Society, Faraday 577 Transactions 1: Physical Chemistry in Condensed Phases. 5 (1989) 1049-1063.

578 [51] Hinchliffe, A., Nikolaidi, B., Soscún Machado, H.J. Density functional studies of the dipole 579 polarizabilities of substituted stilbene, azoarene and related push-pull molecules. International 580 Journal of Molecular Sciences. 8 (2004) 224-238.

581 [52] Tehrani-Bagha, A.R., Holmberg, K. Solubilization of hydrophobic dyes in surfactant 582 solutions. Materials. 2 (2013) 580-608.

583 [53] Foroudian, H.J., Gillitt, N.D., Bunton, C.A. Effects of nonionic micelles on 584 dephosphorylation and aromatic nucleophilic substitution. Journal of colloid and interface 585 science. 1 (2002) 230-237.

586 [54] Brown, J.M., Elliott, R.L., Griggs, C.G., Helmchen, G., Nill, G. Selective Micellar Catalysis 587 with Histidinyl Surfactants of Defined Absolute Configuration. Angewandte Chemie 588 International Edition in English. 10 (1981) 890-892.

589 [55] Benko, J. The measurement of molecular weight of lignosulf onic acids and related 590 materials by diffusion. Tappi. 47 (1964) 508-514.

591 [56] Lindström, T. The colloidal behaviour of kraft lignin. Colloid and Polymer Science. 2 592 (1980) 168-173. 
593 [57] Sarkanen, S., Teller, D.C., Stevens, C.R., McCarthy, J.L. Lignin. 20. Associative 594 interactions between kraft lignin components. Macromolecules. 12 (1984) 2588-2597.

595 [58] Sarkanen, S., Teller, D.C., Hall, J., McCarthy, J.L. Lignin. 18. Associative effects among 596 organosolv lignin components. Macromolecules. 2 (1981) 426-434.

597 [59] Deng, Y., Feng, X., Zhou, M., Qian, Y., Yu, H., Qiu, X. Investigation of aggregation and 598 assembly of alkali lignin using iodine as a probe. Biomacromolecules. 4 (2011) 1116-1125. 


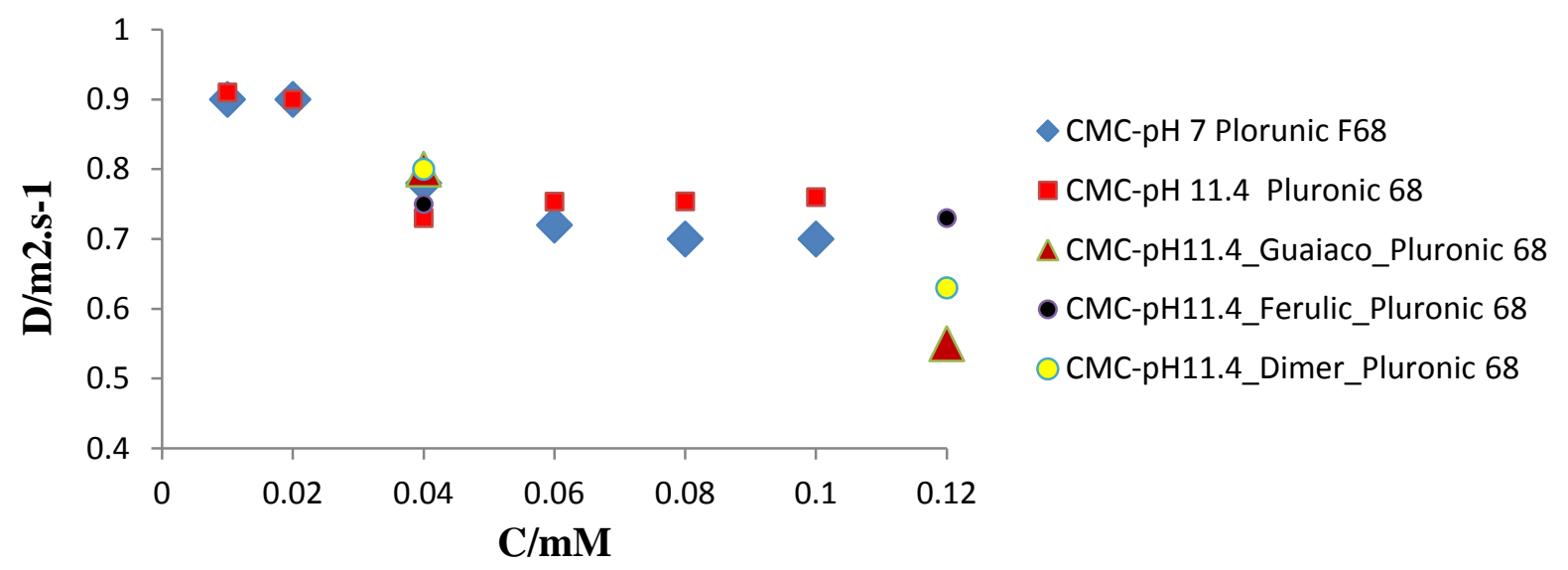

Fig. 1.
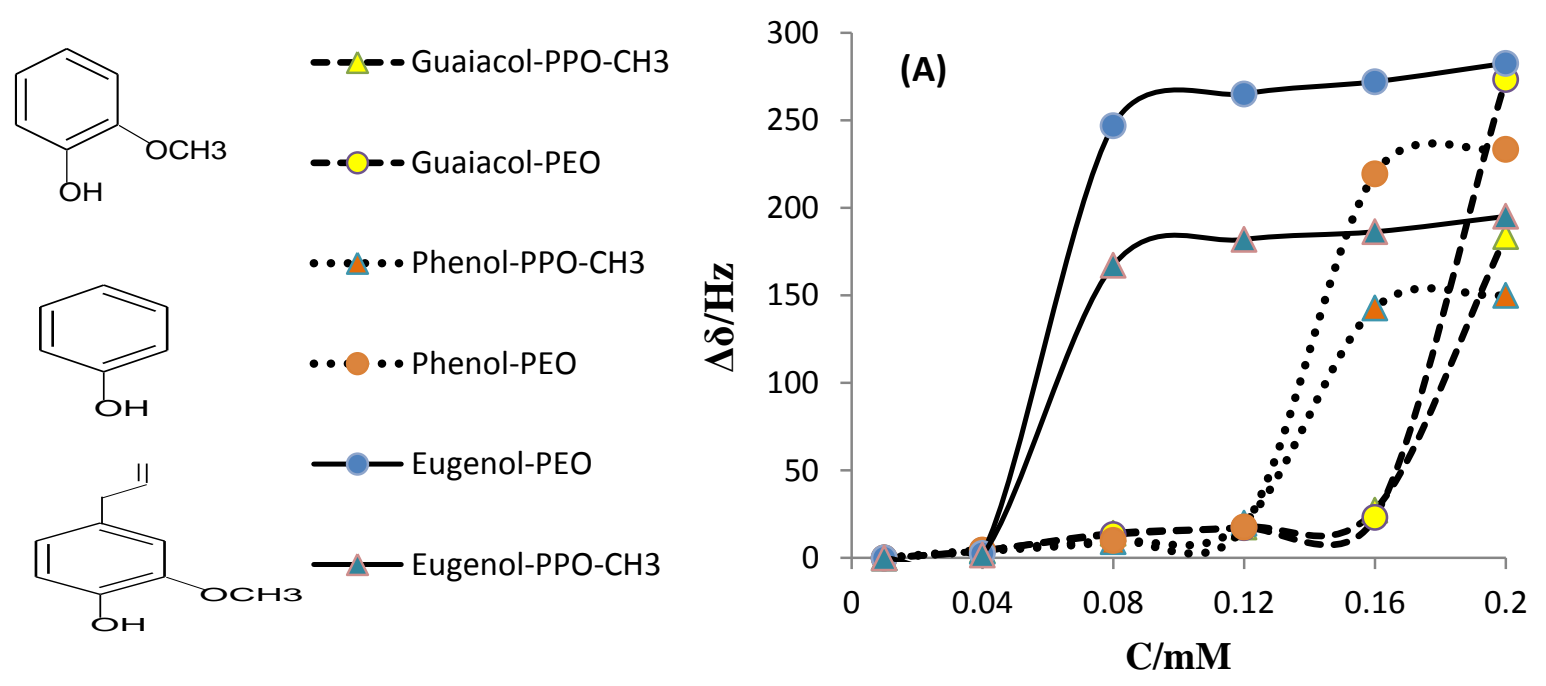

Fig. 2. (a) 


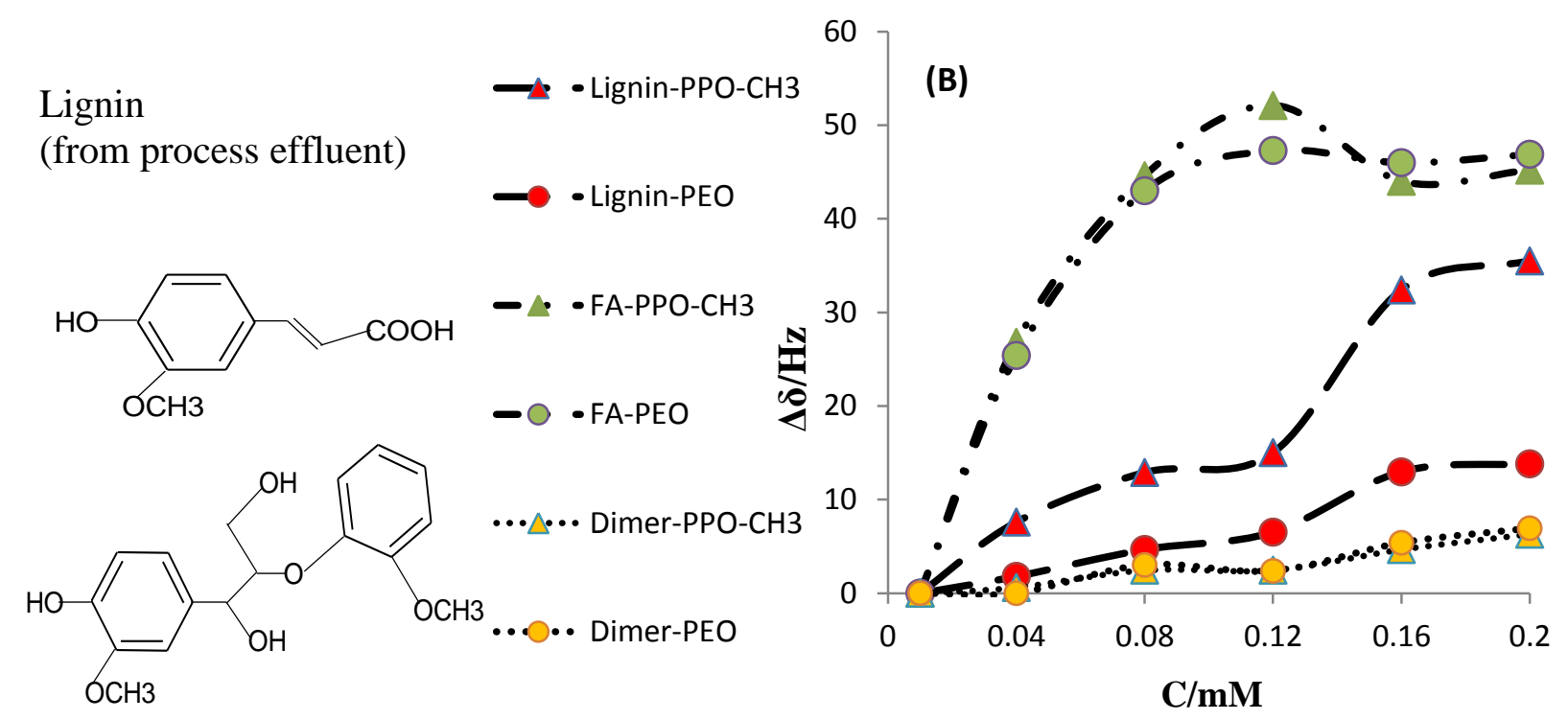

Fig. 2. (b) 


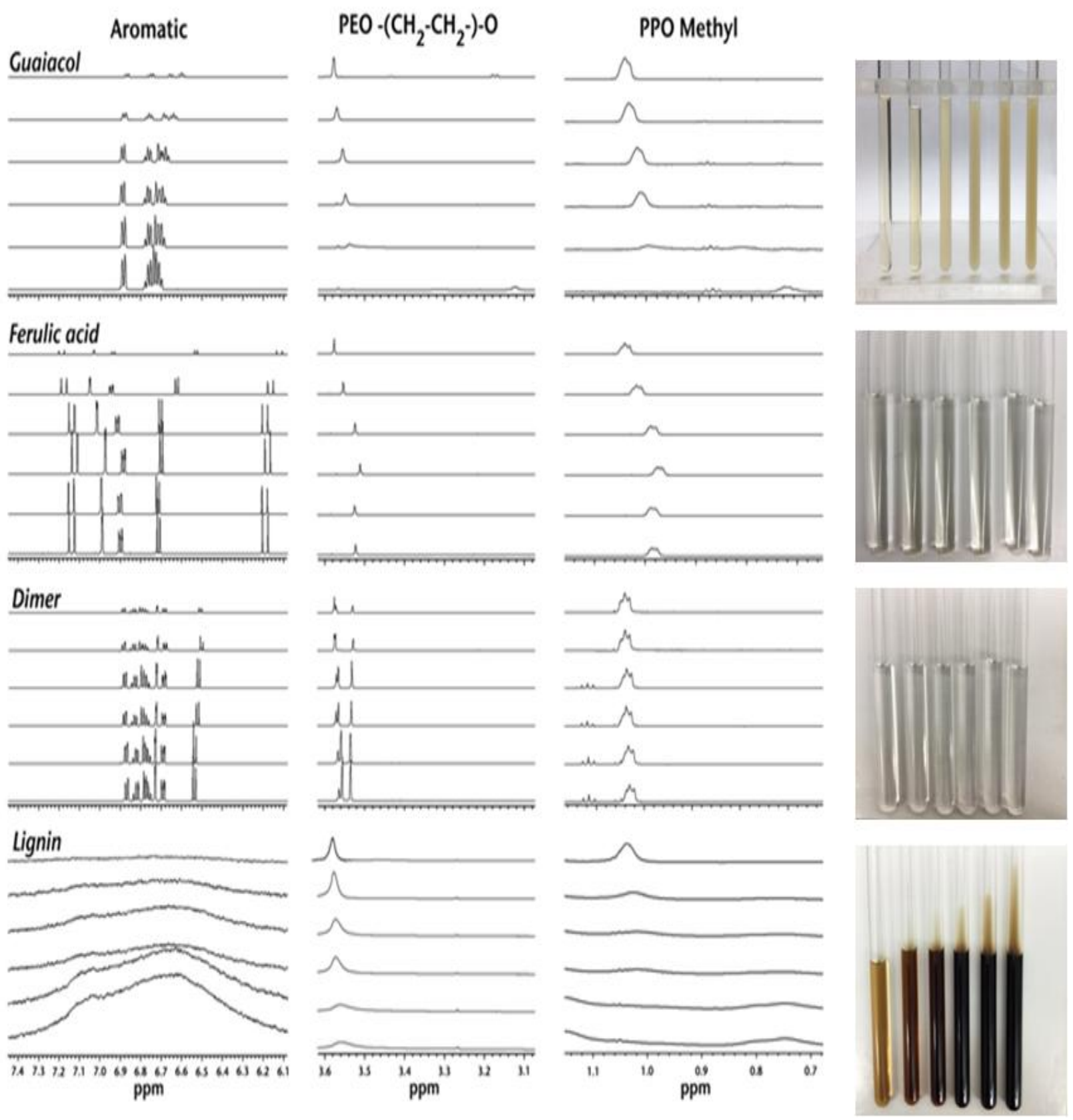

Fig. 3. 


\section{Figure caption}

Scheme1. Process flowchart

Fig. 1. Diffusion coefficient changes of Pluronic F-68 ABA block copolymer as a function of various concentrations of the block copolymer $(0.01,0.02,0.04,0.06,0.08,0.1,0.12 \mathrm{mM}, \mathrm{pH}$ 11.4 and 7) and addition of guaiacol, guaiacylglycerol-beta-guaiacyl ether (dimer), and ferulic acid guests (each $0.04 \mathrm{mM}$ ) at the CMC. The final data set on the right shows only the effect of addition of guaiacol, dimer, and ferulic acid (each $0.12 \mathrm{mM}$ ) to the polymer solution at its CMC $(0.04 \mathrm{mM})$ at $\mathrm{pH} 11.4$.

Fig. 2. (a) Graph of chemical shift changes $(\Delta \delta / \mathrm{Hz})$ for $\mathrm{PEO}-\left(\mathrm{CH}_{2}-\mathrm{CH}_{2}\right)$ and $\mathrm{PO}-\left(\mathrm{CH}_{3}\right)$ of Pluronic F-68 block copolymer $(0.04 \mathrm{mM}, \mathrm{pH} 11.4)$ as a function of various concentrations $(\mathrm{C} / \mathrm{mM})$ of guaiacol, phenol and eugenol.

Fig. 2. (b) Graph of chemical shift changes $(\Delta \delta)$ for PEO- $\left(\mathrm{CH}_{2}-\mathrm{CH}_{2}\right)$ and $\mathrm{PPO}-\left(\mathrm{CH}_{3}\right)$ of Pluronic F-68 block copolymer $(0.04 \mathrm{mM}, \mathrm{pH} 11.4)$ as a function of various concentrations $(\mathrm{C} / \mathrm{mM})$ of lignin, ferulic acid and dimer.

Fig. 3. ${ }^{1} \mathrm{H}$ NMR stacked plots of guaiacol, ferulic acid, guaiacylglycerol-beta-guaiacyl ether (dimer) and lignin with concentrations $0.01-0.20 \mathrm{mM}$ (from top line to bottom line) in Pluronic F68- $\mathrm{D}_{2} \mathrm{O}$ solution with $0.04 \mathrm{mM}$ concentration and $\mathrm{pH} 11.4$ (See the supplementary data). 


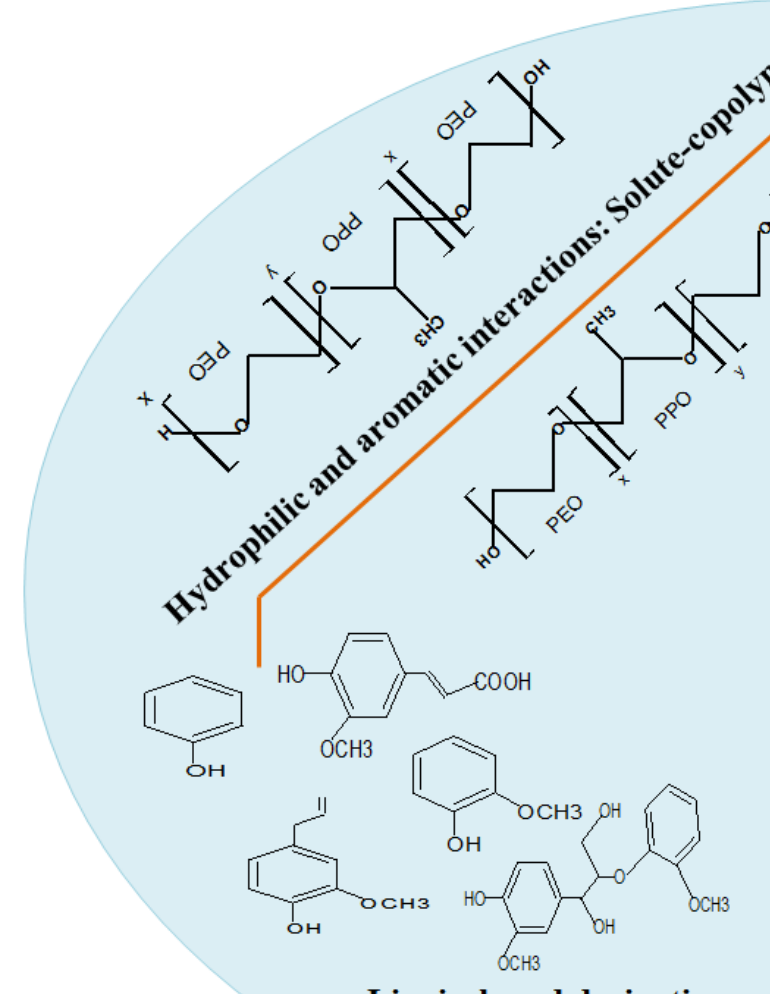

Lignin-based derivatives

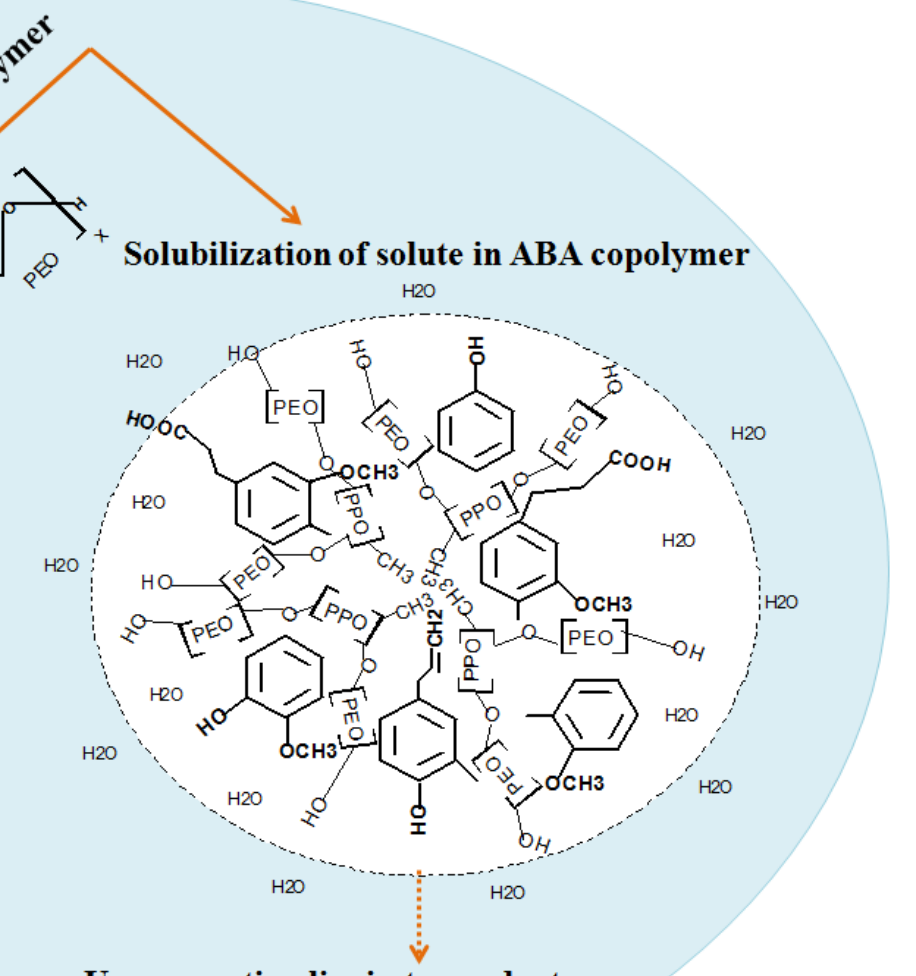

Up-converting lignin to products 